\title{
Catechol-O-Methyltransferase Polymorphisms and Some Implications for Cognitive Therapeutics
}

\author{
Catherine M. Diaz-Asper, Daniel R. Weinberger, and Terry E. Goldberg \\ Clinical Brain Disorders Branch, National Institute of Mental Health, National Institutes of Health, Bethesda, Maryland 20892
}

\begin{abstract}
Catechol-O-methyltransferase (COMT) is a gene involved in the degradation of dopamine and may both increase susceptibility to develop schizophrenia and affect neuronal functions involved in working memory. A common variant of the COMT gene (val ${ }^{108 / 158}$ met) has been widely reported to affect prefrontally mediated working memory function, with the high-activity val allele associated with poorest performance across a number of tests sensitive to updating and target detection. Pharmacological manipulations of COMT val ${ }^{108 / 158}$ met also have reliably produced alterations in cognitive function, in line with an inverted $U$ function of prefrontal dopamine signaling. Furthermore, there is accumulating evidence that COMT val ${ }^{108 / 158}$ met genotype may influence the cognitive
\end{abstract}

response to antipsychotic treatment in schizophrenia patients, with met allele load predicting the greatest improvement with medication. Recently, other single-nucleotide polymorphisms (SNPs) across the COMT gene have emerged as possible risk alleles for schizophrenia, although little is known about whether they affect prefrontal cognition in a manner similar to COMT val ${ }^{108 / 158}$ met. Preliminary evidence suggests a modest role for a SNP in the 5' region of the gene on select tests of attention and target detection. Haplotype effects also may account for a modest percentage of the variance in test performance, and are an important area for future study. Key Words: Catechol-O-methyltransferase, COMT, working memory, executive function, prefrontal cognition.

\section{INTRODUCTION}

Working memory dysfunction is widely considered a cardinal feature of schizophrenia ${ }^{1,2}$ and appears to be a heritable phenotype related to risk for developing the disorder. $^{3-8}$ One gene that affects neuronal functions involved in working memory, and may be a potential susceptibility gene to develop schizophrenia, is catecholO-methyltransferase (COMT), a methylation enzyme that converts dopamine to inactive 3-methoxytyramine. ${ }^{9,10}$ COMT exists in both membrane-bound (MB) and soluble (S) forms, which differ by a 50 -amino acid signal anchor in the MB form. S-COMT predominates in peripheral human tissue, whereas MB-COMT is expressed primarily in the brain. ${ }^{9,11}$ Although MB-COMT is expressed throughout the brain, its functional effects appear regionally specific. It is expressed primarily in neurons and appears more abundant in prefrontal cortex and hippocampus than in striatum or brainstem dopamine neurons. ${ }^{12}$ In fact, pharmacological studies have

Address correspondence and reprint requests to Terry E. Goldberg, Ph.D., Professor of Psychiatry, Albert Einstein College of Medicine, Director, Neurocognition, Division of Psychiatry Research, Zucker Hillside Hospital, 75-59 263rd Street, Glen Oaks, NY 11004. demonstrated that the catabolic flux of synaptic dopamine through the COMT pathway exceeds $60 \%$ in the prefrontal cortex, compared with only $15 \%$ in the striatum. ${ }^{13}$ The seemingly selective effect of COMT on dopamine signaling in prefrontal regions does not reflect the distribution of COMT per se, but rather, the relative low abundance of synaptic dopamine transporters found in prefrontal cortex, which are critically involved in the inactivation of synaptic dopamine in striatum. ${ }^{14}$ Consistent with this, studies of COMT knockout mice have demonstrated increased dopamine levels only in the prefrontal cortex, in addition to enhanced memory function. $^{15-17}$

\section{THE COMT VAL ${ }^{108 / 158}$ MET POLYMORPHISM}

The COMT gene, mapped to chromosome 22q11, contains a functional polymorphism, val ${ }^{108 / 158}$ met $(r s 4680)$, that results in two common variants of the enzyme (val and met) (see FIG. 1). The amino acid change affects the stability of the enzyme, such that the val form has $\sim 40 \%$ greater enzyme activity, and therefore greater dopamine degradation, than the met form (with both S- and MBCOMT affected). ${ }^{18,19}$ Although findings lack consen- 


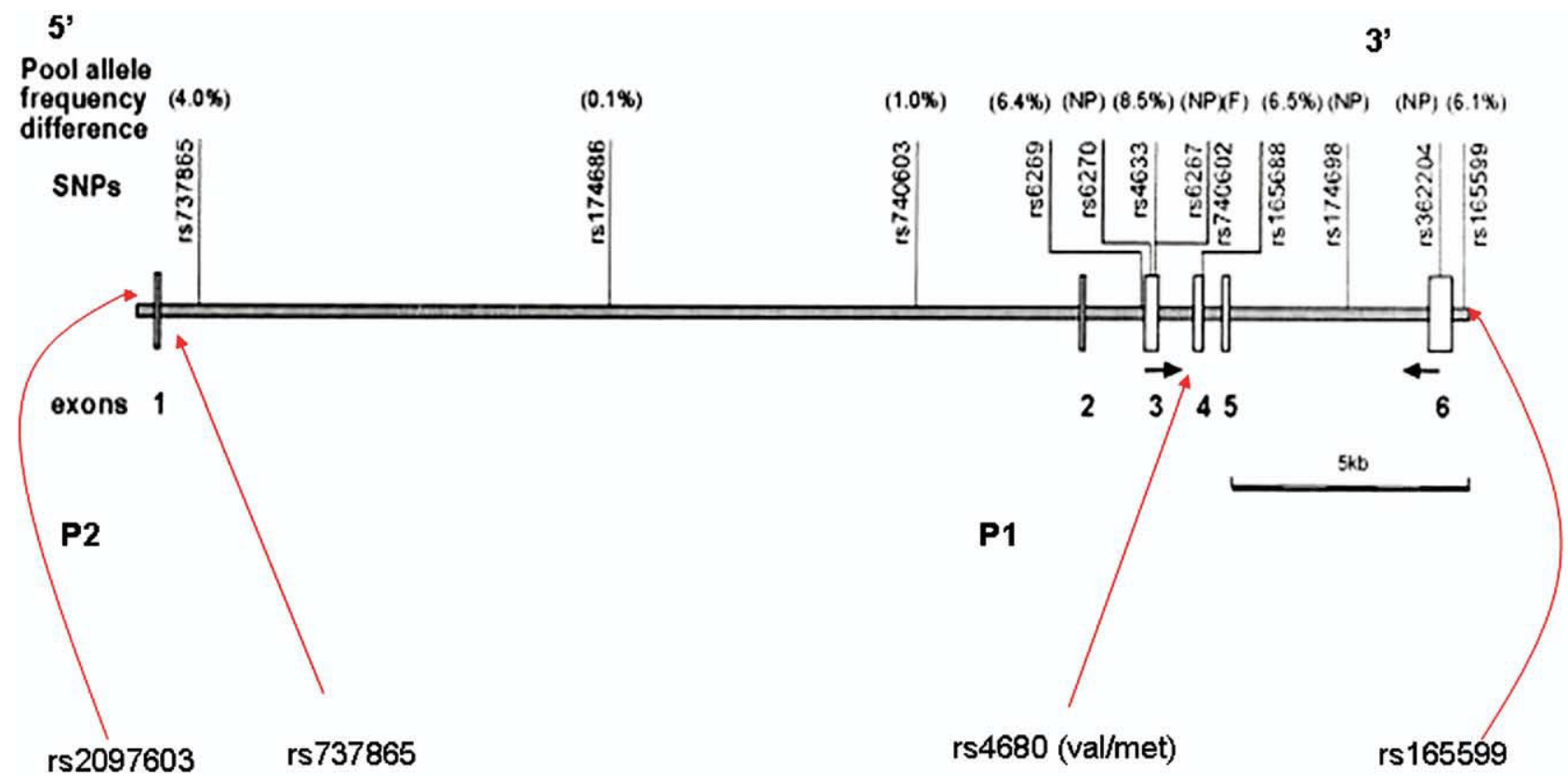

FIG. 1. The COMT gene.

sus, ${ }^{20-22}$ several family-based studies suggest preferential transmission of the val allele in schizophrenia. ${ }^{8,23-25}$ Furthermore, this polymorphism putatively affects dopamine-regulated prefrontal cortical activity during working memory tasks. ${ }^{8,26-32}$ The hypothesis is that COMT is important in the modulation of prefrontal dopamine, and the high-activity val form of the allele, results in hypodopaminergia, increasing both risk for schizophrenia and impairments in prefrontally mediated working memory functions.

\section{EFFECTS ON PREFRONTAL COGNITION}

In only a relatively few years, a number of studies have reported that the COMT val ${ }^{108 / 158}$ met polymorphism affects working memory function. For example, our group previously reported that this genotype predicts performance on two different tests of working memory/ executive function [the Wisconsin Card Sorting Test (WCST) and N-BACK], with the high-activity val allele being associated with poorest performance. ${ }^{8,28}$ These effects were observed independent of psychiatric diagnosis, and genotype was not associated with IQ or other nonprefrontally mediated cognitive tasks. Egan et al. ${ }^{8}$ reported that $\sim 4 \%$ of the variance in perseverative errors on the WCST was explained by COMT val ${ }^{108 / 158}$ met genotype. Furthermore, val allele loading predicted reduced efficiency of the dorsolateral prefrontal cortex during the N-BACK working memory task using functional magnetic resonance imaging (fMRI). ${ }^{28}$ Specifically, met allele load predicted a more efficient physiological response (less BOLD activation) in prefrontal cortex in the 2-BACK condition of the task.
Goldberg and colleagues ${ }^{28}$ used the N-BACK task to examine the effect of COMT val ${ }^{108 / 158}$ met and genetic risk for schizophrenia on load, delay, and updating subprocesses of working memory. Results revealed similar effects of COMT val ${ }^{108 / 158}$ met genotype on 1 - and 2-BACK accuracy and reaction time, with val homozygotes performing most poorly and met homozygotes performing the best. Given the lack of differential effects across the two levels of the task, the authors concluded that COMT val ${ }^{108 / 158}$ met genotype specifically affects information updating and ordering in the face of competing stimuli, reflected in demands for continuous selection and deselection of targets that are common to both the 1-BACK and 2-BACK.

There have been several independent replications of these findings. For example, Malhotra and colleagues ${ }^{30}$ administered the WCST to 73 healthy volunteers and reported that met homozygotes committed significantly fewer perseverative errors than val allele carriers. ${ }^{29,32}$ Additionally, Gallinat et al. ${ }^{27}$ reported that an event related potential (ERP) during a P300 "oddball" task was affected by COMT val ${ }^{108 / 158}$ met, with amplitude in frontal regions lower in met homozygotes than val carriers, consistent with the notion that the met allele is associated with less prefrontal physiological noise. A larger EEG study by Winterer et al. ${ }^{33}$ found again that COMT val alleles predicted poorer signal to noise in prefrontal cortex during the P300 evoked response, and the odds ratio of this effect in male subjects was 3:4. Furthermore, a recent study examined the effect of the genotype on prefrontally mediated cognition in healthy children and reported that the val allele was associated with impaired 


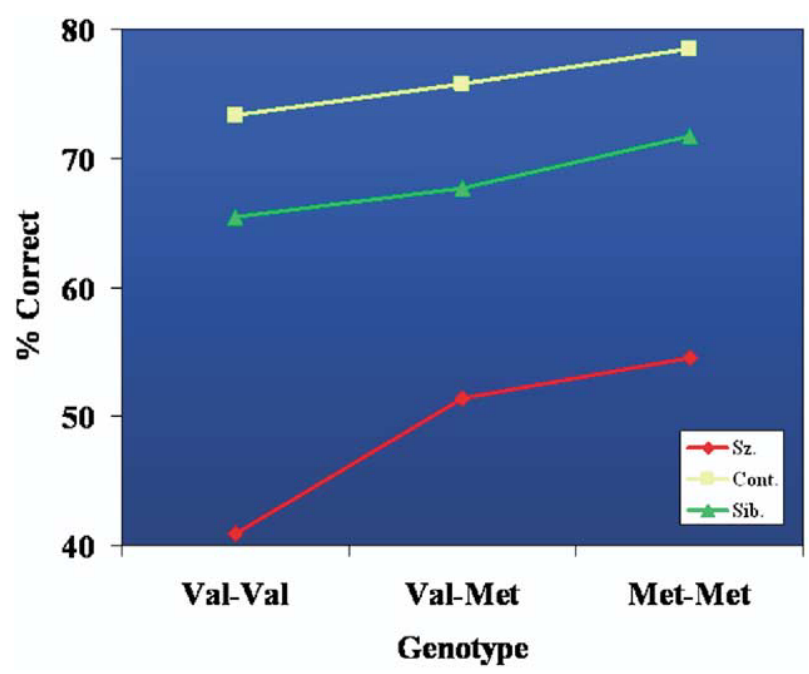

FIG. 2. Percent correct on the 2-BACK working memory task, as a function of COMT val ${ }^{108 / 158}$ met genotype in schizophrenic patients, their siblings, and healthy control subjects.

performance on a "spatial" Stroop task requiring working memory and inhibition. ${ }^{34}$ Bilder and colleagues ${ }^{26}$ studied the effects of COMT val ${ }^{108 / 158}$ met in 58 chronic schizophrenic patients, on a set of 15 neuropsychological tests, reduced to four cognitive domain scores. Results revealed that the met allele was associated with superior performance in the domain of attention and processing speed, raising the possibility that COMT genotype may be associated with a somewhat broader range of cognitive functions than simply working memory.

We recently examined the effect of COMT genotype on a broad range of working memory/executive function tests, in a large sample of schizophrenic probands $(\mathrm{n}=$ $325)$, their nonpsychotic siblings $(\mathrm{n}=359)$, and normal control subjects $(\mathrm{n}=330) .{ }^{35}$ We replicated and extended our earlier report of a COMT val ${ }^{108 / 158}$ met genotype effect on working memory function ${ }^{28}$ because individuals with two copies of the val allele performed most poorly on the N-BACK working memory test, irrespective of psychiatric diagnosis. Met homozygotes demonstrated the best performance on the task, and heterozygotes performed intermediately between the other two groups (FIG. 2). Once again, we failed to see a COMT/ diagnosis interaction on any condition of the N-BACK, in the face of a significant COMT effect, which suggests that normal controls, siblings, and probands were more or less equally affected by COMT val ${ }^{108 / 158}$ met. The fact that the three diagnostic groups were affected in a near linear manner in this, and our earlier studies, ${ }^{28}$ supports the notion of an additive genetic model in which allele loading influences working memory in a similar manner, irrespective of an individual's specific background.

However, in contrast with our previous findings, we found a significant COMT val ${ }^{108 / 158}$ met genotype effect on 0-BACK performance in our recent study. Addition- ally, we found a significant COMT val ${ }^{108 / 158}$ met effect on performance on the Distractibility portion of the Continuous Performance Test (CPT) (FIG. 3), which we did not report previously with our smaller sample. ${ }^{28}$ Both the 0 -BACK and the CPT place demands on attention, vigilance, and encoding in the service of target detection, and results of an exploratory factor analysis indicated that both load on the same factor (and presumably share similar underlying cognitive mechanisms). To consider these results further, we developed a novel attention regulation task that involved selective attention to increasingly complex global or local elements of a figure and control of interfering contingencies, and found that COMT val ${ }^{108 / 158}$ met genotype predicted performance on this task and efficiency of the cingulate cortex response measured with fMRI. ${ }^{36}$ Again, the val allele was associated with more errors on the high-load version of the task, and greater cingulate cortex engagement. Our results are at least partially consistent with those of Bilder et al. ${ }^{26}$ and suggest that COMT val ${ }^{108 / 158}$ met exerts an effect even on these attentional control or target detection functions, raising the possibility that the genotype may be responsible for a broader category of cognition than was previously thought. Certainly, there is evidence supporting a role for dopamine in attention and target detection tasks. ${ }^{37-39}$

Our recent study also served to extend the results of our previous work by reporting an apparently diseasespecific COMT val ${ }^{108 / 158}$ met effect on a componential test of attentional set-shifting, the Intradimensional/Extradimensional (ID/ED) task. We found a significant COMT/diagnosis interaction on two of the three ID/ED measures examined. Probands with two copies of the val allele committed substantially more errors at a relatively early stage of the test (the Compound Discrimination

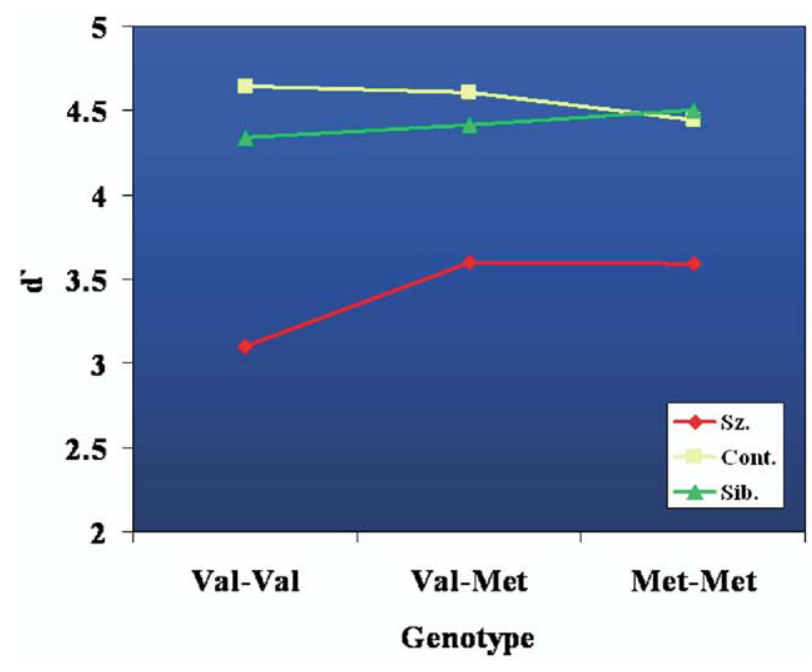

FIG. 3. Accuracy $\left(d^{\text {c}}\right)$ on the Distractibility portion of the Continuous Performance Test, as a function of COMT val ${ }^{108 / 158}$ met genotype in schizophrenic patients, their siblings, and healthy control subjects. 


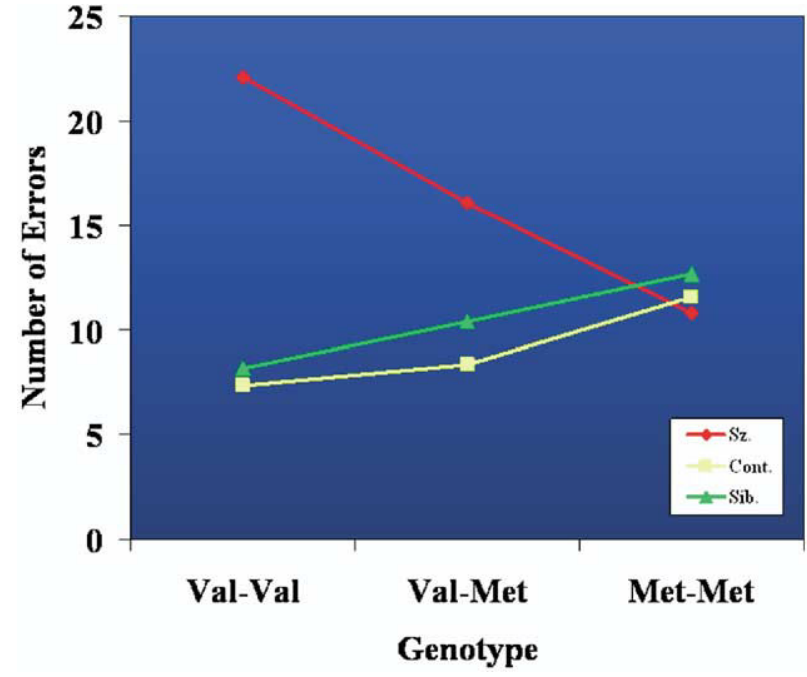

FIG. 4. Number of Compound Discrimination errors on the ID/ED task, as a function of COMT val ${ }^{108 / 158}$ met genotype in schizophrenic patients, their siblings, and healthy control subjects.

stage) than probands with one or no copies of the allele (FIG. 4). Distracting and irrelevant stimuli are introduced at this stage, and subjects must ignore stimuli from the novel dimension and continue to respond to the previously rewarded stimulus. Our results are consistent with those of Crofts et al., ${ }^{37}$ who reported significant deficits at a similar stage of acquiring a series of compound visual discriminations in marmosets with catecholamine depletions in the frontal cortex. The fact that we saw substantial group effects at this stage also is consistent with our earlier reports of a differential deficit among probands at this stage of the task. ${ }^{40} \mathrm{We}$ also found a COMT/diagnosis interaction at a later, more complex stage of the task (the Intradimensional Shift stage), which suggests probands with two copies of the val allele were significantly less able to discern the conceptual category within which they were responding. A deficit in Intradimensional Shifting represents an impairment in the ability to generalize a discrimination learned for a particular set of exemplars to another set from the same category in the face of newly salient distractors. One could argue that this type of rule learning requires the updating and ordering of information in the face of competing stimuli, akin to the sort of cognitive operation required to perform the N-BACK. Our recent results therefore raise the possibility that COMT val ${ }^{108 / 158}$ met genotype affects both updating or rule learning during interference-laden contexts (ID/ED, N-BACK) and attentional control (CPT, 0-BACK), with inheritance of the val allele associated with poorest performance. However, these findings of significant COMT/diagnosis interactions on the ID/ED task must be tempered by the fact that they may reflect a ceiling effect in the healthy control and sibling groups.
Although we found a significant group effect at the Extradimensional Shift stage of the ID/ED task, we failed to see a COMT/diagnosis interaction, which was unexpected, given that Extradimensional Shifting is the core component of the WCST, and is the basis for achieving novel sorting categories. ${ }^{40} \mathrm{We}$, and others, have previously reported a significant COMT val ${ }^{108 / 158}$ met effect on WCST performance, ${ }^{28,30,41}$ so we expected to replicate this result in our recent study. However, we failed to see a significant COMT effect or COMT/diagnosis interaction for the WCST. These findings, using a substantially larger sample than in our previous study, argue that the WCST may not be the ideal task to examine COMT effects on prefrontal working memory function-rather, the Compound Discrimination and Intradimensional Shift stages of the ID/ED task may be more sensitive measures to detect the specific cognitive operations influenced by COMT val $^{108 / 158}$ met genotype.

Finally, consistent with our previous work, ${ }^{28}$ we failed to see a COMT val ${ }^{108 / 158}$ met genotype effect on intelligence, as assessed by the four-subtest version of the Wechsler Adult Intelligence Scale-Revised (WAIS-R). Arguably, certain subtests of the WAIS-R (e.g., Arithmetic) make limited demands on attention and working memory, yet these demands were presumably insufficient to observe a COMT effect, despite a considerably larger sample size than our previous study. This relative specificity for prefrontal cognition is consistent with basic animal research ${ }^{15}$ showing that, although COMT is expressed throughout much of anterior cortex, its function appears especially relevant for prefrontal dopaminergic signaling.

\section{NEGATIVE FINDINGS}

In summary, these findings support the contention that COMT val ${ }^{108 / 158}$ met genotype influences prefrontally mediated working memory/executive function, with inheritance of two copies of the val allele most detrimental to performance. Nevertheless, results from other studies have not been uniformly positive. For example, Tsai and colleagues $^{41}$ reported no association between COMT $\mathrm{val}^{108 / 158}$ met genotype and performance on the WCST, and Stefanis et al. ${ }^{42}$ found no relationship between COMT val ${ }^{108 / 158}$ met and performance on either the CPT or N-BACK tasks in normal subjects. More recently, Ho and colleagues ${ }^{43}$ reported no significant COMT $\mathrm{val}^{108 / 158}$ met genotype or COMT/diagnosis effects on select tests of working memory, including the WCST and backward digit span. Further research is likely required to resolve these discrepant findings. However, the methodological differences between studies may, at least in part, explain some of the discrepancies. In terms of subject variables, both the Tsai et al. ${ }^{41}$ and Stefanis et al. ${ }^{42}$ studies used relatively ho- 
mogeneous populations (young Chinese women and young Greek men, respectively), and the frequency distribution of the met allele in the Tsai et al. ${ }^{41}$ study was atypical for Caucasion American subjects, possibly resulting in lack of power to detect differences. Ho et al. $^{43}$ tested young, mildly impaired patients who were early in the course of their illness (35\% who were neuroleptic naive). Some proportion of variability between studies may also be attributable to differences in the cognitive tasks used. For example, a number of different versions of the N-BACK exist, and differ both in complexity of task demands, and response requirements. Our version of the N-BACK places significant demands upon maintenance and updating (like other versions of the N-BACK), yet additionally requires a constant response to every stimulus displayed. From their description, it is unclear which version of the N-BACK that Stefanis and colleagues ${ }^{42}$ used. Of note, both Tsai et al. ${ }^{41}$ and Ho et al. ${ }^{43}$ reported no effect of COMT genotype on WCST performance, as did we, again arguing that this may not be the optimal indicator of working memory function to use in genetic association studies.

Others have argued that inconsistent findings between studies are attributable to task differences in terms of tonic and phasic dopamine signaling. This position holds that the low activity met allele is of benefit during tasks of cognitive stability requiring tonic dopamine activation, but detrimental on tasks of cognitive flexibility requiring phasic activation. ${ }^{44,45}$ For example, Stefanis and colleagues ${ }^{45}$ recently reported that met genotype loading was associated with reduced variability in reaction time on a continuous performance test, suggesting greater performance stability. Whereas val and met alleles appear to vary in terms of the tasks that they advantage, it is highly speculative whether these differences can be explained by differential effects of phasic or tonic dopamine signaling.

\section{PHARMACOLOGICAL MANIPULATION}

Pharmacological manipulations of COMT also have reliably produced alterations in cognitive function. For example, Mattay et al. ${ }^{31}$ reported that, in val homozygotes, amphetamine enhanced the efficiency of prefrontal cortical function during a working memory task, assayed with fMRI. In contrast, met homozygotes (who tended to have superior baseline prefrontal function) demonstrated no amphetamine effect at low-to-moderate working memory loads, but a deterioration in cortical efficiency at high working memory loads. These data are consistent with accumulating evidence favoring an inverted $U$ functional-response curve to increasing dopamine signaling in the prefrontal cortex. ${ }^{46,47}$

The COMT inhibitor tolcapone also has been associ- ated with improvements in working memory function, in both humans ${ }^{48,49}$ and rats. ${ }^{50,51}$ Although tolcapone appears primarily to affect peripheral COMT, it does penetrate the blood-brain barrier ${ }^{52}$ and inhibits brain COMT activity in vivo. ${ }^{53,54}$ In rodent behavioral studies, administration of tolcapone after pretraining facilitated spatial working memory performance. ${ }^{50,51}$ Furthermore, Gasparini and colleagues ${ }^{49}$ assessed the effect of tolcapone on cognitive function in eight patients with advanced Parkinson's disease, and reported that over a 6-month period, patients significantly improved on measures of attention, verbal short-term memory, visuospatial recall, constructional praxia, and motor symptoms. Interestingly, this drug had not been studied in healthy individuals as a cognitive-enhancing agent. To address this, we recently examined whether tolcapone would improve working memory/executive function in normal control subjects. ${ }^{55}$ Specifically, we predicted that increased prefrontal efficiency associated with tolcapone would be most pronounced in val homozygotes, with likely a worsening in met homozygotes, in line with the proposed inverted $\mathrm{U}$ function of prefrontal dopamine signaling. ${ }^{46}$ In a double-blind, placebo-controlled crossover study, 47 healthy controls (including 15 val homozygotes and 11 met homozygotes) were administered $200 \mathrm{mg}$ of tolcapone or placebo three times a day for 7 days. ${ }^{55}$ Each subject completed a battery of tests sensitive to prefrontal cognitive function, at the conclusion of each arm of the protocol. In the main, results obtained supported our hypotheses. Significant and near-significant drug effects were found on two tests of working memory (TrailMaking Part B and N-BACK). Additionally, we found significant tolcapone/COMT interactions on a test of episodic memory, and the later Intradimensional Shift stage of the ID/ED task. These interactions were characterized by improved performance for val homozygotes on tolcapone, yet worsening performance for met homozygotes on tolcapone. Several other tests thought to engage prefrontal circuitry did not demonstrate these effects, including the WCST, letter-number sequencing, and verbal fluency. Nevertheless, the results suggest that COMT val $^{108 / 158}$ met genotype may predict the normal human cognitive response to COMT inhibition. Tolcapone in particular may prove to be a valuable tool for improving prefrontal function in individuals homozygous for the val allele.

\section{RESPONSE TO ANTIPSYCHOTICS}

There is mounting evidence that COMT val ${ }^{108 / 158}$ met genotype may influence the cognitive response to antipsychotic medications in schizophrenic patients. ${ }^{56-59}$ Recently, Bertolino et al. ${ }^{57}$ reported that the genotype predicts working memory performance and activation of the dorsolateral prefrontal cortex in a group of schizo- 
phrenic patients after 8 weeks of olanzapine treatment. Specifically, met allele load predicted improvement in prefrontal physiology, and on the N-BACK working memory task, as assessed with fMRI. Val homozygotes demonstrated no such improvement. Weickert et al. ${ }^{58}$ reported remarkably similar findings in their examination of the association between COMT val ${ }^{108 / 158}$ met genotype and cognitive response to antipsychotic medications in patients with schizophrenia. Twenty inpatients were administered a battery of cognitive tasks, once after 4 weeks of antipsychotic medication, and once after 4 weeks of placebo administration, in a counterbalanced, double-blind, within-subject design. Results revealed significant post-treatment gains in working memory for met homozygotes only. Specifically, met homozygotes displayed enhanced N-BACK performance during administration of antipsychotic medication relative to their own performance during the placebo control period. In contrast, val homozygotes demonstrated no such improvement and performed significantly more poorly than met homozygotes. The observed effects appeared independent of specific antipsychotic medications.

\section{OTHER COMT SINGLE-NUCLEOTIDE POLYMORPHISMS}

Recently, other single-nucleotide polymorphisms (SNPs) across the COMT gene have emerged as possible alleles that increase risk for schizophrenia, including $r s 737865$ (located in intron 1), rs 165599 (located in the $3^{\prime}$ flanking region), and $r s 2097603$ (in the $\mathrm{P} 2$ promoter region) ${ }^{60-63}$ (see FIG. 1). For example, Shifman and colleagues ${ }^{63}$ tested several SNPs and haplotypes in the COMT gene and reported that two common SNPs, one upstream (rs737865) and the other in or near the $3^{\prime}$ untranslated region (rs165599), were associated with schizophrenia in a large sample of Israeli Ashkenasi Jews. They reported that, although COMT val ${ }^{108 / 158}$ met bears only a modest risk effect on schizophrenia, haplotypes containing either or both of these other risk alleles in combination with $\mathrm{val}^{108 / 158}$ met contribute significantly to disease risk, especially in women. The $\mathrm{G} / \mathrm{G}$ allele appeared most associated with risk to develop schizophrenia. $\mathrm{Li}$ et al. ${ }^{64}$ examined seven SNP markers in 198 family trios from Southwest China, and reported nonsignificant preferential transmission of the rs2097603 A allele in schizophrenia. At present, the influence of these specific SNPs on COMT enzyme activity, prefrontal cortical activity and cognitive function, is not well understood. A recent study by Chen et al. ${ }^{18}$ reported that $r s 737865$, and $r s 165599$, had little or no effect on COMT mRNA expression levels, protein immunoreactivity, or enzyme activity in postmortem human prefrontal cortical tissue, arguing a lack of functionality of these SNPs, but evidence of effects of val ${ }^{108 / 158}$ met and $r s 2097603$ enzyme activity were found. However, to our knowledge, no one has yet examined in a large group of probands, siblings, and healthy controls whether these SNPs affect working memory/executive function in a manner similar to COMT val ${ }^{108 / 158}$ met. We recently addressed this, using the same large sample of probands, siblings, and controls as was described above. ${ }^{35}$

\section{RS737865 (5' REGION)}

Because $r s 737865$ is in linkage disequilibrium with $r s 4680$ (COMT val ${ }^{108 / 158}$ met), analyses were conducted for $r s 737865$ against COMT val ${ }^{108 / 158}$ met homozygote backgrounds. Overall, findings from this SNP were far less compelling than we reported for COMT val $^{108 / 158}$ met. For example, in contrast to the findings with COMT val ${ }^{108 / 158}$ met, an effect of $r s 737865$ genotype was seen only on 0-BACK performance, and only when analyzed against a met-met background. Similarly, main effects of $r s 737865$ were seen on both subtests of the CPT and at the early Compound Discrimination stage of the ID/ED task, but only against a met-met background. The only test on which a main effect of $r s 737865$ genotype, and a COMT/diagnosis interaction, was observed against both COMT val-met homozygote backgrounds was the Distractibility portion of the CPT. (Interestingly, the observed effects for these tests were in the expected direction, with probands homozygous for the $\mathrm{G}$ allele performing most poorly overall).

The fact that the majority of significant findings with this SNP were observed against one homozygote background, but not the other, could be interpreted in a number of different ways. One interpretation is that this represents the effects of a haplotype. Another view is that the two homozygote backgrounds should be considered as two independent samples, such that one result is a cross-validation of the other (given that the same individuals cannot be included in both analyses). The fact that we failed to see such a cross-validation on the majority of tests with $r s 737865$ would tend to argue against the reliability of these findings. The other, most conservative position, would be that our findings are spurious, and simply the result of multiple comparisons within the data. The correct interpretation of these findings will most likely have to await the results of further studies.

\section{RS165599 (3' REGION) AND RS2097603 (P2 PROMOTER REGION)}

We failed to see any significant COMT main effects on working memory/executive function for either the rs165599 or $r s 2097603$ SNPs, aside from a main effect of rs 165599 on later Intradimensional Shift errors on the ID/ED task, against a met homozygote background only. These results contrast with a very small study of Chinese 


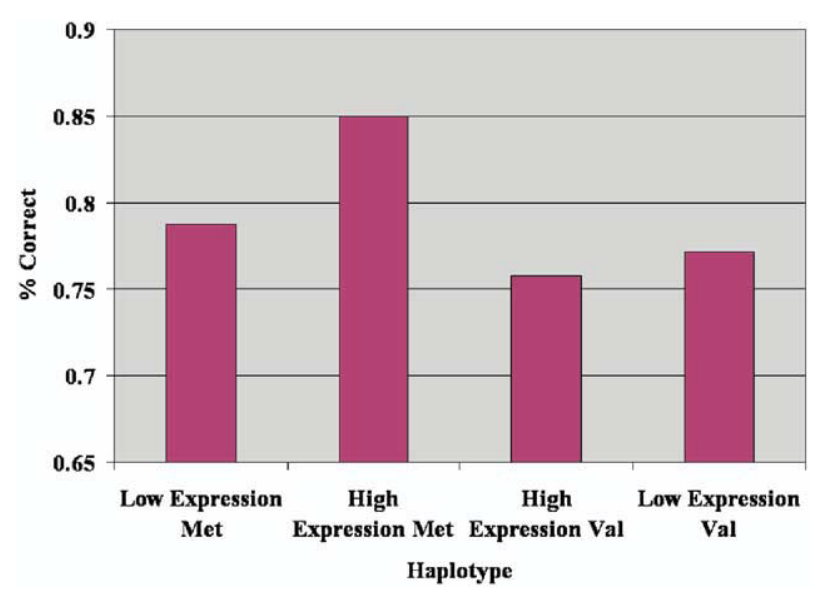

FIG. 5. Percent correct on the 1-BACK working memory task, as a function of COMT P2 promoter/val ${ }^{108 / 158}$ met haplotype.

patients recently conducted by Chan and colleagues, ${ }^{65}$ in which more pronounced memory and executive function deficits were seen in patients with the $r s 165599 \mathrm{G} / \mathrm{G}$ genotype. Again, further studies, using large samples, will need to address this apparent discrepancy. We did find an interaction effect of $r s 2097603$ and diagnosis on both subtests of the CPT, with the A/A allele appearing most disadvantageous for probands. Interestingly, the A allele was reported in one study to be (nonsignificantly) preferentially transmitted in schizophrenia. ${ }^{64}$ Significant interactions between genotype and gender were also noted for various tests, as were complex three-way interactions, but the small sample size in some cells rendered interpretation of these results difficult.

Interestingly, and in support of our recent findings, Weickert et al. $^{58}$ reported no effect of $r s 737865$, $r s 165599$, or $r s 2097603$ on working memory function in a small sample of probands when on versus off neuroleptic medication. The possibility still exists, however, that these SNPs do affect working memory function, but our very small cell sizes for some critical analyses may have resulted in insufficient power to detect differences.

\section{HAPLOTYPE EFFECTS}

It is important to note that some of the previously reported effects of COMT genotype on cognition may have been subtly impacted by expression differences due to promoter SNPs. Therefore, we recently examined the effect of the P2 promoter/ val ${ }^{108 / 158}$ met haplotype on prefrontally mediated cognitive function, ${ }^{35}$ finding that results were in the expected direction on select tests that have reliably been found to be sensitive to COMT effects (e.g., 1-BACK) (FIG. 5), but not on other tests (e.g., WCST). On this basis, we concluded that haplotype effects likely account for a modest percentage of the variance in test performance (less than 1\%) after COMT $\mathrm{val}^{108 / 158}$ met genotype effects have been explained.
Shifman et al. ${ }^{63}$ argued that COMT val ${ }^{108 / 158}$ met alone bore only a minor, if any, effect on schizophrenia risk but may have accorded greater risk when correlated with $r$ 737865, which has a significant effect on schizophrenia susceptibility. Their findings further argued that COMT shows a stronger association with schizophrenia in females, with rs 165599 affecting primarily women, and $r 5737865$ affecting both genders, but in different ways. Our recent findings certainly illustrated complex interactions between COMT genotype and gender on some tests, and are open to several different interpretations. In general, however, we did not find strong evidence favoring the notion that females demonstrate greater sensitivity to COMT than their male counterparts. ${ }^{35}$

In conclusion, findings from our group and others demonstrate that COMT val ${ }^{108 / 158}$ met genotype affects prefrontally mediated working memory function, specifically updating and attentional set-shifting in the face of interference. Inheritance of two copies of the val allele appears most disadvantageous to task performance. We also found modest support for an effect of rs737865 genotype on select tests of attention and target detection, with the G/G allele most disadvantageous to performance. However, we found virtually no evidence for a COMT effect on working memory in two other SNPs across the COMT gene, suggesting that, although these SNPs may increase susceptibility to develop schizophrenia, they do not appear to contribute to working memory/ executive dysfunction to the same extent as COMT val $^{108 / 158}$ met. $^{35}$

\section{CONCLUSIONS}

There is now considerable evidence that a common variant in the COMT gene (val ${ }^{108 / 158}$ met) accounts for significant variance in prefrontally mediated cognitive function, specifically on tests of target detection and updating. Pharmacological manipulations of COMT also have reliably been associated with alterations in prefrontally mediated cognitive function, and the genotype also may influence the cognitive response to antipsychotic medications in schizophrenic patients. Preliminary evidence also suggests a modest role for another SNP in the COMT gene (rs737865) on select tests of attention and target detection. Results reviewed here reveal that COMT not only plays a role in susceptibility for schizophrenia, but also delineates a relationship between an intermediate phenotype (working memory dysfunction) and a related genotype.

\section{REFERENCES}

1. Goldberg TE, Green MF. Neurocognitive functioning in patients with schizophrenia: an overview. In: Psychopharmacology: the fifth generation of progress (Davis KL, ed). New York: Raven Press, 2002. 
2. Weickert TW, Goldberg TE, Gold JM, Bigelow LB, Egan MF, Weinberger DR. Cognitive impairments in patients with schizophrenia displaying preserved and compromised intellect. Arch Gen Psychiatry 57:907-913, 2000.

3. Franke P, Maier W, Hain C, Klingler T. Wisconsin Card Sorting Test: an indicator of vulnerability to schizophrenia? Schizophr Res 6:243-249, 1992.

4. Yurgelun-Todd D, Kinney DK. Patterns of neuropsychological deficits that discriminate schizophrenic individuals from siblings and control subjects. J Neuropsychiatry Clin Neurosci 5:294-300, 1993.

5. Faraone S, Seidman LJ, Kremen WS, Pepple JR, Lyons MJ, Tsuang MT. Neuropsychological functioning among the nonpsychotic relatives of schizophrenic patients: a diagnostic efficiency analysis. J Abnormal Psychol 104:286-304, 1995.

6. Goldberg TE, Torrey EF, Gold JM, Bigelow LB, Ragland RD, Taylor E, et al. Genetic risk of neuropsychological impairment in schizophrenia: a study of monozygotic twins discordant and concordant for the disorder. Schizophr Res 17:77-84, 1995.

7. Cannon TD, Huttunen MO, Lonnqvist J, Tuulio-Henriksson A, Pirkola T, Glahn D, et al. The inheritance of neuropsychological dysfunction in twins discordant for schizophrenia. Am J Hum Genet 67:369-382, 2000.

8. Egan MF, Goldberg TE, Kolachana BS, Callicott JH, Mazzanti CM., Straub RE, et al. Effect of COMT Val108/158Met genotype on frontal lobe function and risk for schizophrenia. Proc Natl Acad Sci USA 98:6917-6922, 2001.

9. Harrison PJ, Weinberger DR. Schizophrenia genes, gene expression, and neuropathology: on the matter of their convergence. Mol Psychiatry 10:40-68, 2004.

10. Weinberger DR, Egan MF, Bertolino A, Callicott JH, Mattay VS, Lipska BK, et al. Prefrontal neurons and the genetics of schizophrenia. Biol Psychiatry 50:825-844, 2001.

11. Tenhunen J, Salminen M, Lundstr"m K, Kiviluoto T, Savolainen $\mathrm{R}$, Ulmanen I. Genomic organization of the human catechol-Omethyltransferase gene and its expression from two distinct promoters. Eur J Biochem 223:1049-1059, 1994.

12. Matsumoto M, Weickert CS, Akil M, Lipska BK, Hyde TM, Herman MM, et al. Catechol O-methyltransferase mRNA expression in human and rat brain: evidence for a role in cortical neuronal function. Neuroscience 116:127-137, 2003.

13. Karoum F, Chrapusta S, Egan MF. 3-Methoxytryptamine is the major metabolite of released dopamine in the rat frontal cortex: reassessment of the effects of antipsychotics on the dynamics of dopamine release and metabolism in the frontal cortex, nucleus accumbens, and striatum by a simple two pool model. J Neurochem 63:972-979, 1994.

14. Sesack SR, Hawrylak VA, Matus C, Guido MA, Levey AI. Dopamine axon varicosities in the prelimbic division of the rat prefrontal cortex exhibit sparse immunoreactivity for the dopamine transporter. J Neurosci 18:2697-2708, 1998.

15. Gogos JA, Morgan M, Luine V, Santha M, Ogawa S, Pfaff D, et al. Catechol-O-methyltransferase-deficient mice exhibit sexually dimorphic changes in catecholamine levels and behavior. Proc Natl Acad Sci USA 95:9991-9996, 1998.

16. Huotari M, Gogos JA, Karayiorgou M, Koponen I, Forsberg M, Raasmaja A, et al. Brain catecholamine metabolism in catecholO-methyltransferase (COMT)-deficient mice. Eur J Neurosci 15: 246-256, 2002.

17. Kneavel M, Gogos J, Karayiorgou K, Luine V. Interaction of COMT gene deletion and environment on cognition. Soc Neurosci Abstract 26:1-2, 2000.

18. Chen J, Lipska BK, Halim N, Ma QD, Matsumoto M, Melhem S, et al. Functional analysis of genetic variation in catechol-O-methyltransferase (COMT): Effects on mRNA, protein, and enzyme activity in postmortem human brain. Am J Hum Genet 75:807821, 2004.

19. Weinshilboum RM, Otterness DM, Szumlanski CL. Methylation pharmacogenetics: catechol O-methyltransferase, thiopurine methyltransferase, and histamine N-methyltransferase. Annu Rev Pharmacol Tox 39:19-52, 1999.

20. Fan JB, Zhang CS, Gu NF, Li XW, Sun WW, Wang HY, et al. Catechol-O-methyltransferase gene $\mathrm{Val} / \mathrm{Met}$ functional polymor- phism and risk of schizophrenia: a large-scale association study plus meta-analysis. Biol Psychiatry 57:139-144, 2005.

21. Glatt SJ, Faraone SV, Tsuang MT. Association between a functional catechol-O-methyltransferase gene polymorphism and schizophrenia: meta-analyses of case-control and family-based studies. Am J Psychiatry 160:469-476, 2003.

22. Munafo MR, Bowes L, Clark TG, Flint J. Lack of association of the COMT (Val(158/108) Met) gene and schizophrenia: a metaanalysis of case-control studies. Mol Psychiatry 10:765-770, 2005.

23. Chen X, Wang X, O'Neill AF, Walsh D, Kendler KS. Variants on the catechol-O-methyltransferase (COMT) gene are associated with schizophrenia in Irish high-density families. Mol Psychiatry 9:962-967, 2004.

24. Kunugi H, Vallada HP, Sham PC, Hoda F, Arranz MJ, Li T, et al. Catechol-O-methyltransferase polymorphisms and schizophrenia: a transmission disequilibrium study in multiply affected families. Psychiatr Genet 7:97-101, 1997.

25. Li T, Sham PC, Vallada H, Xie T, Tang X, Murray RM, et al. Preferential transmission of the high activity allele of COMT in schizophrenia. Psychiatr Genet 6:131-133, 1996.

26. Bilder RM, Volavka J, Czobor P, Malhotra AK, Kennedy JL, Ni $\mathrm{XQ}$, et al. Neurocognitive correlates of the COMT Val158Met polymorphism in chronic schizophrenia. Biol Psychiatry 52:701$707,2002$.

27. Gallinat J, Bajbouj M, Sander T, Schlattmann P, Xu K, Ferro EF, et al. Association of the G1947A COMT (Val108/158Met) gene polymorphism with prefrontal $\mathrm{P} 300$ during information processing. Biol Psychiatry 54:40-48, 2003.

28. Goldberg TE, Egan MF, Gscheidle T, Coppola R, Weickert T, Kolachana BS, et al. Executive subprocesses in working memory: relationship to catechol-O-methyltransferase $\mathrm{Val}{ }^{158} \mathrm{Met}$ genotype and schizophrenia. Arch Gen Psychiatry 60:889-896, 2003.

29. Joober R, Gauthier J, Lal S, Bloom D, Lalonde P, Rouleau G, et al. Catechol-O-methyltransferase val ${ }^{158}$ met gene variants associated with performance on the Wisconsin Card Sorting Test. Arch Gen Psychiatry 59:662-663, 2002.

30. Malhotra AK, Kestler LJ, Mazzanti C, Bates JA, Goldberg T, Goldman D. A functional polymorphism in the COMT gene and performance on a test of prefrontal cognition. Am J Psychiatry 159:652-654, 2002

31. Mattay VS, Goldberg TE, Fera F, Hariri AR, Tessitore A, Egan MF, et al. Catechol-O-methyltransferase val ${ }^{158}$ met genotype and individual variation in the brain response to amphetamine. Proc Natl Acad Sci USA 100:6186-6191, 2003.

32. Rosa A, Peralta V, Cuesta MJ, Zarzuela A, Serrano F, MartinezLarrea A, et al. New evidence of association between COMT gene and prefrontal neurocognitive function in healthy individuals and from sibling pairs discordant for psychosis. Am J Psychiatry 161: 1110-1112, 2004.

33. Winterer G, Coppola R, Goldberg TE, Egan MF, Jones DW, Sanchez CE, Weinberger DR. Prefrontal broadband noise, working memory, and genetic risk for schizophrenia. Am J Psychiatry 161: 490-500, 2004.

34. Diamond A, Briand L, Fossella J, Gehlbach L. Genetic and neurochemical modulation of prefrontal cognitive functions in children. Am J Psychiatry 161:125-132, 2004.

35. Diaz-Asper CM, Goldberg TE, Kolachana BS, Straub RE, Egan $\mathrm{MF}$, Weinberger DR. Genetic variation in catechol-O-methyltransferase (COMT): effects on working memory function in schizophrenic patients and healthy controls. In preparation.

36. Blasi G, Mattay VS, Bertolino A, Elvevaag B, Callicott JH, Das S, et al. Effect of catechol-O-methyltransferase val ${ }^{158}$ met genotype on attentional control. J Neurosci 25:5038-5045, 2005.

37. Crofts HS, Dalley JW, Collins P, Van Denderen JCM, Everitt BJ, Robbins TW, et al. Differential effects of 6-OHDA lesions of the frontal cortex and caudate nucleus on the ability to acquire an attentional set. Cereb Cortex 11:1015-1026, 2001.

38. Eisenberg J, Mei-Tal G, Steinberg A, Tartakovsky E, Zohar A, Gritsenko I, et al. Haplotype relative risk study of catechol-Omethyltransferase (COMT) and attention deficit hyperactivity disorder (ADHD): association of the high-enzyme activity val allele with ADHD impulsive-hyperactive phenotype. Am J Med Genet 88:497-502, 1999. 
39. Nieoullon A. Dopamine and the regulation of cognition and attention. Prog Neurobiol 67:53-83, 2002.

40. Jazbec S, Pantelis C, Weickert T, Robbins T, Weinberger D, Goldberg T. IDED performance in schizophrenia: impact of distraction and perseveration. In preparation.

41. Tsai SJ, Yu YW, Chen TJ, Chen JY, Liou YJ, Chen MC, et al. Association study of a functional catechol-O-methyltransferasegene polymorphism and cognitive function in healthy females. Neurosci Lett 338:123-126, 2003.

42. Stefanis NC, Van Os J, Avramopoulos D, Smyrnis N, Evdokimidis I, Hantoumi I, et al. Variation in catechol-O-methyltransferase val ${ }^{158}$ met genotype associated with schizotypy but not cognition: a population study in 543 young men. Biol Psychiatry 56:510-515, 2004.

43. Ho BC, Wassink TH, O'Leary DS, Sheffield VC, Andreasen NC. Catechol-O-methyltransferase val(158)met gene polymorphism in schizophrenia: working memory, frontal lobe MRI morphology and frontal cerebral blood flow. Mol Psychiatry 10:287-298, 2005.

44. Nolan KA, Bilder RM, Lachman HM, Volavka J. Catecholo-Omethyltransferase val ${ }^{158}$ met polymorphism in schizophrenia: differential effects of val and met alleles on cognitive stability and flexibility. Am J Psychiatry 161:359-361, 2004.

45. Stefanis NC, van Os J, Avramopoulos D, Smyrnis N, Evdokimidis I, Stefanis CN. Effect of COMT val ${ }^{158}$ met polymorphism on the Continuous Performance Test, Identical Pairs version: tuning rather than improving performance. Am J Psychiatry 162:17521754, 2005.

46. Goldman-Rakic PS. The cortical dopamine system: role in memory and cognition. Adv Pharmacol 42:707-711, 1998.

47. Lidow MS, Koh PO, Arnsten AF. D1 dopamine receptor in the mouse prefrontal cortex: immunocytochemical and cognitive neuropharmacological analyses. Synapse 47:101-108, 2003.

48. Stahl SM. Finding what you are not looking for: strategies for developing novel treatments in psychiatry. NeuroRx 3:7-9, 2006.

49. Gasparini M, Fabrizio E, Bonifati V, Meco G. Cognitive improvement during Tolcapone treatment in Parkinson's disease. J Neural Trans 104:887-894, 1997.

50. Khromova I, Rauhala P, Zolotov N, Mannisto PT. Tolcapone, an inhibitor of catechol O-methyltransferase, counteracts memory deficits caused by bilateral cholinotoxin lesions of the basal nuclei of Meynert. Neuroreport 6:1219-1222, 1995.

51. Liljequist R, Haapalinna A, Ahlander M, Li YH, Mannisto PT. Catechol O-methyltransferase inhibitor tolcapone has minor influence on performance in experimental memory models in rats. Behav Brain Res 82:195-202, 1997.

52. Ceravolo R, Piccini P, Bailey DL, Jorga KM, Bryson H, Brooks DJ. 18F-dopa PET evidence that tolcapone acts as a central COMT inhibitor in Parkinson's disease. Synapse 43:201-207, 2002.
53. Da Prada M, Zurcher G, Kettler R, Colzi A. New therapeutic strategies in Parkinson's disease: inhibition of MAO-B by Ro196327 and of COMT by Ro40-7592. New York: Plenum Press, 1991.

54. Zurcher G, Dingemanse J, Da Prada M. Ro-40-7592, a potent inhibitor of extracerebral and brain catechol-O-methyltransferase: preclinical and clinical findings. Rome: John Libbey S. R. L., 1991.

55. Apud JA, Mattay V, Chen J, Kolachana BS, Callicott JH, Rasetti $\mathrm{R}$, et al. Tolcapone improves cognition and cortical information processing in normal human subjects. In preparation.

56. Blasi G, Bertolino A. Imaging genomics and response to treatment with antipsychotics in schizophrenia. NeuroRx 3:117-130, 2006.

57. Bertolino A, Caforio G, Blasi G, De Candia M, Latorre V, Petruzzella V, et al. Interaction of COMT (Val(108/158)Met) genotype and olanzapine treatment on prefrontal cortical function in patients with schizophrenia. Am J Psychiatry 161:1798-1805, 2004.

58. Weickert TW, Goldberg TE, Mishara A, Apud JA, Kolachana BS, Egan MF, et al. COMT val108/158met genotype predicts working memory response to antipsychotic medications. Biol Psychiatry 56:677-682, 2004.

59. Kapurt S, Agid O, Mizrahi R, Li M. How antipsychotics workfrom receptors to reality. NeuroRx 3:000-000, 2006.

60. Bray NJ, Buckland PR, Williams NM, Williams HJ, Norton N, Owen MJ, et al. A haplotype implicated in schizophrenia susceptibility is associated with reduced COMT expression in human brain. Am J Hum Genet 73:152-161, 2003.

61. DeMille MMC, Kidd JR, Ruggeri V, Palmatier MA, Goldman D, Odunsi A, et al. Population variation in linkage disequilibrium across the COMT gene considering promoter region and coding region variation. Hum Genet 111:521-537, 2002.

62. Palmatier MA, Pakstis AJ, Speed W, Paschou P, Goldman D, Odunsi A, et al. COMT haplotypes suggest $\mathrm{P} 2$ promoter region relevance for schizophrenia. Mol Psychiatry 9:859-870, 2004.

63. Shifman S, Bronstein M, Sternfeld M, Pisante-Shalom A, LevLehman E, Weizman A, et al. A highly significant association between a COMT haplotype and schizophrenia. Am J Hum Genet 71:1296-1302, 2002.

64. Li T, Ball D, Zhao J, Murray RM, Liu X, Sham PC, et al. Familybased linkage disequilibrium mapping using SNP marker haplotypes: application to a potential locus for schizophrenia at chromosome 22q11. Mol Psychiatry 5:77-84, 2000.

65. Chan RCK, Chen RYL, Chen EYH, Hui TCK, Cheung EFC, Cheung HK, et al. The differential clinical and neurocognitive profiles of COMT SNP rs 165599 genotypes in schizophrenia. J Int Neuropsychol Soc 11:202-204, 2005. 